\title{
Detection of Citrobacter koseri carrying beta-lactamase KPC-2 in a hospitalised patient, Greece, July 2011
}

A Mavroidii ${ }^{1}$ I Neonakis ${ }^{1,2}$, A Liakopoulos $^{1}$, A Papaioannou $^{1}$, M Ntala $^{3}$, F Tryposkiadis $^{3}$, V Miriagou $^{4}$,

E Petinaki (petinaki@med.uth.gr) ${ }^{1}$

1. Department of Microbiology, University Hospital of Larissa, Greece

2. Department of Microbiology, University Hospital of Heraklion, Crete, Greece

3. Department of Cardiology, University Hospital of Larissa, Greece

4. Laboratory of Bacteriology, Hellenic Pasteur Institute, Athens, Greece

Citation style for this article:

Mavroidi A, Neonakis I, Liakopoulos A, Papaioannou A, Ntala M, Tryposkiadis F, Miriagou V, Petinaki E. Detection of Citrobacter koseri carrying beta-lactamase KPC-2 in a hospitalised patient, Greece, July 2011.

Euro Surveill. 2011;16(41):pii=19990. Available online: http://www.eurosurveillance.org/ViewArticle.aspx?Articleld=19990

Article published on 13 October 2011

This report describes the detection of Citrobacter koseri carrying $K$. pneumoniae carbapenemase (KPC2) isolated in July 2011 from a Greek patient, who was also colonised by a Klebsiella pneumoniae strain coproducing KPC-2 and Verona integron-encoded metallo-beta-lactamase (VIM)-1.

\section{Case description}

On July 2011, a Greek male in his early 80 s was admitted to the University Hospital of Larissa, Greece, due to an acute myocardial infarction. He had chronic hypertension due to diabetes and had been hospitalised in the same hospital two months earlier, due to suspicion of tuberculosis, which was later ruled out (sputum and bronchoalveolar lavage cultures were negative for Mycobacterium tuberculosis). His respiratory tract symptoms soon remitted and he was discharged.

On admission for the myocardial infarction in July, he was given an empirical prophylactic antibiotic regimen of amoxicillin and clindamycin. On the fourth day of hospitalisation, he became febrile $\left(40^{\circ} \mathrm{C}\right)$ and cultures taken from various samples (blood, vein catheter, urine, sputum and bronchoalveolar lavage) were examined. Gram staining of the bronchoalveolar lavage showed the presence of Gram-negative rods along with leucocyte infiltration, indicating a respiratory tract infection. The presence of the Gram-negative bacterium was confirmed by culture, while the rest of the clinical samples were negative.

Using the VITEK 2 system (bioMérieux, France), the isolate was found to be Citrobacter koseri with reduced susceptibility or resistance to beta-lactams, including carbapenems (cefotaxime, ceftazidime, cefepime, aztreonam and ertapenem), but susceptible to fluoroquinolones (ciprofloxacin) and aminoglycosides (amikacin and gentamicin) (Table). Identification of $C$. koseri was further confirmed by PCR (amplifying the $16 \mathrm{~S}$ ribosomal (r) RNA) and sequencing [1]. Non-beta-lactam antibiotics, such as quinolones and aminoglycosides, were active against the isolate (Table). The antimicrobial chemotherapy was altered to ciprofloxacin and the fever abated within days. Screening of faecal samples for carbapenem-resistant Enterobacteriaceae performed with McConkey agar supplemented with $1 \mathrm{mg} / \mathrm{L}$ imipenem showed that the patient's gastrointestinal tract was colonised by an imipenem-resistant Klebsiella pneumoniae (Table).

Ten days later and while still on treatment with ciprofloxacin, the patient had a second febrile episode and imipenem-non-susceptible $K$. pneumoniae isolates were recovered from three cultures (two from blood and one from a vein catheter). The antibiotic resistance profiles of these three isolates were similar to that of the $K$. pneumoniae isolate from the faecal sample (Table). The patient was then successfully treated with a combination of tigecycline and gentamicin.

\section{Background}

The emergence of Enterobacteriaceae producing class A beta-lactamases of $K$. pneumoniae carbapenemase (KPC) type is a major clinical and public health concern as these enzymes have the potential to compromise treatment with all beta-lactams, including carbapenems. They are typically transposon-encoded determinants and therefore have the ability to move between plasmids and across bacterial species.

C. koseri, an environmental, Gram-negative bacterium, is occasionally found as a coloniser of the human gastrointestinal tract.* Although the potential virulence of the species is considered low, it is sporadically implicated in serious nosocomial infections [2]. Furthermore, C. koseri has the ability to easily incorporate antibioticresistance determinants [3]. Although there are several reports of $C$. koseri isolates bearing extended-spectrum beta-lactamase (ESBL) genes, the detection of $C$. koseri isolates producing a carbapenemase is rare [4-6]. To our knowledge, there is only one report of a C. koseri isolate producing Verona integron-encoded 
metallo-beta-lactamase VIM-(1) [7]. Here, we present the first report of a $C$. koseri clinical isolate producing KPC-2.

\section{Laboratory investigations}

All five bacterial strains isolated from the patient (one C. koseri and four K. pneumoniae) were studied further. The minimum inhibitory concentration of various antiobiotics were determined by Etest (bioMérieux, France) and the results were interpreted according to the criteria of the European Committee on Antimicrobial Susceptibility Testing [8]. The modified Hodge test was used for phenotypic detection of carbapenemase production of either KPC or VIM type: all five isolates were positive. Meropenem-boronate and meropenemethylenediaminetetraacetic acid (EDTA) combined disk tests, performed as described elsewhere [9], indicated the presence of a KPC in the $C$. koseri isolate and the co-production of KPC and metallo-beta-lactamase $(\mathrm{MBL})$ in the $K$. pneumoniae isolates.

PCR assays were used to screen all five isolates for bla genes encoding KPC, VIM, SHV, TEM, OXA-1, CMY and CTX-M [10-12]. Sequencing of the entire amplified genes revealed the carriage of $b / a_{\text {TEM-1 }}$ and $b l a_{\mathrm{KPC}-2}$ by C. koseri and $b l a_{\mathrm{TEM}-1}, b l a_{\mathrm{KPC}-2}$, and $b l a_{\mathrm{VIM}-1}$ by K. pneumoniae. No nucleotide differences were observed in the $b l a_{\mathrm{TEM}-1}$ and $b l a_{\mathrm{KPC}-2}$ genes (which are known to be highly conserved) in the $C$. koseri and $K$. pneumoniae isolates.

The genetic relatedness of the $K$. pneumoniae isolates was investigated by multilocus sequence typing based on seven housekeeping genes (rpoB, gapA, $m d h, p g i$, phoE, infB and tonB) [13]. All four isolates were found to belong to ST147.

It was not possible to determine whether $C$. koseri could be found in the patient's gastrointestinal tract. Detection of C. koseri in faecal samples relies on amplification of $16 \mathrm{~S}$ rRNA by PCR; however, this would amplify almost all bacteria that colonise the gastrointestinal tract.

\section{Control measures taken}

As recommended by the Greek health authorities, patients are not screened for carbapenem-resistant bacteria on admission or during hospitalisation. Weekly carrier screening is performed only in wards where a carbapenemase-producing bacterium is isolated.

When C. koseri was isolated from the patient, faecal samples were taken from the other six patients who were being treated in the same unit. None were found to be colonised by a KPC-positive Enterobacteriaceae. The patient was isolated in a single-bed room, under strict infection control measures.

\section{Discussion}

Previous studies have presented evidence for horizontal dissemination of $b a_{\mathrm{KPC}}$ genes among enterobacterial species worldwide [14-17]. However, to our knowledge, this is the first report of $C$. koseri producing KPC-2.

Enterobacteriaceae producing KPC were introduced in Greece in 2007 [18]. Since then, the prevalence of these microorganisms has risen to epidemic proportions, especially in teaching hospitals in the main urban areas [19]. This can be attributed to the overcrowded patient population in these hospitals, serious shortage of specialised personnel and consequently inadequate infection control. In the hospital in Larissa, where the prevalence of KPC-positive $K$. pneumoniae is approximately $27 \%$, similar to that in other teaching hospitals in Greece [20], isolates of various species, such as Serratia marcencens, Escherichia coli and Enterobacter aerogenes carrying bla $_{\mathrm{KPC}}$ genes have been occasionally isolated from infected or colonised patients.

TABLE

Minimum inhibitory concentrations of various antimicrobial agents against isolated bacteria from a hospitalised patient, Greece, July 2011

\begin{tabular}{|c|c|c|c|c|c|c|c|c|c|c|c|c|}
\hline \multirow{2}{*}{ Isolate (type of sample) } & \multicolumn{12}{|c|}{ Etest results for the antimicrobial agents $\left(\mathrm{mg} / \mathrm{L}\right.$ and interpretation ${ }^{\mathrm{a}}$ ) } \\
\hline & IMP & MEM & ERT & CTX & CAZ & FEP & ATM & $\mathrm{COL}$ & TIG & CIP & GM & AN \\
\hline $\begin{array}{l}\text { Citrobacter koseri } \\
\text { (bronchoalveolar lavage) } \\
n=1\end{array}$ & $\begin{array}{c}0.75 \\
S\end{array}$ & $\begin{array}{c}0.19 \\
\mathrm{~S}\end{array}$ & $\begin{array}{l}8 \\
R\end{array}$ & $\begin{array}{l}4 \\
\mathrm{R}\end{array}$ & $\begin{array}{c}2 \\
\text { NS }\end{array}$ & $\begin{array}{l}1.5 \\
\text { NS }\end{array}$ & $\begin{array}{l}4 \\
\mathrm{R}\end{array}$ & $\begin{array}{c}0.25 \\
\mathrm{~S}\end{array}$ & $\begin{array}{c}0.25 \\
\mathrm{~S}\end{array}$ & $\begin{array}{c}0.25 \\
\mathrm{~S}\end{array}$ & $\begin{array}{c}0.19 \\
S\end{array}$ & $\begin{array}{l}2 \\
S\end{array}$ \\
\hline $\begin{array}{l}\text { Klebsiella pneumoniae } \\
\text { (blood) } \\
n=2\end{array}$ & $\begin{array}{c}32 \\
R\end{array}$ & $\begin{array}{c}16 \\
R\end{array}$ & $\begin{array}{c}32 \\
\mathrm{R}\end{array}$ & $\begin{array}{c}64 \\
R\end{array}$ & $\begin{array}{c}256 \\
\mathrm{R}\end{array}$ & $\begin{array}{c}32 \\
R\end{array}$ & $\begin{array}{c}64 \\
R\end{array}$ & $\begin{array}{c}16 \\
R\end{array}$ & $\begin{array}{c}0.5 \\
S\end{array}$ & $\begin{array}{c}16 \\
R\end{array}$ & $\begin{array}{l}1 \\
S\end{array}$ & $\begin{array}{c}12 \\
\text { NS }\end{array}$ \\
\hline $\begin{array}{l}\text { K. pneumoniae } \\
\text { (vein catheter) } \\
\mathrm{n}=1\end{array}$ & $\begin{array}{c}32 \\
R\end{array}$ & $\begin{array}{c}16 \\
R\end{array}$ & $\begin{array}{c}32 \\
R\end{array}$ & $\begin{array}{c}64 \\
R\end{array}$ & $\begin{array}{c}256 \\
R\end{array}$ & $\begin{array}{c}32 \\
R\end{array}$ & $\begin{array}{c}64 \\
R\end{array}$ & $\begin{array}{c}16 \\
R\end{array}$ & $\begin{array}{c}0.5 \\
S\end{array}$ & $\begin{array}{c}16 \\
R\end{array}$ & $\begin{array}{l}1 \\
S\end{array}$ & $\begin{array}{c}12 \\
\text { NS }\end{array}$ \\
\hline $\begin{array}{l}\text { K. pneumoniae } \\
\text { (faecal) } \\
\mathrm{n}=1\end{array}$ & $\begin{array}{c}32 \\
\mathrm{R}\end{array}$ & $\begin{array}{c}16 \\
R\end{array}$ & $\begin{array}{c}32 \\
\mathrm{R}\end{array}$ & $\begin{array}{c}64 \\
R\end{array}$ & $\begin{array}{c}256 \\
\mathrm{R}\end{array}$ & $\begin{array}{c}32 \\
\mathrm{R}\end{array}$ & $\begin{array}{c}64 \\
R\end{array}$ & $\begin{array}{c}16 \\
R\end{array}$ & $\begin{array}{c}0.5 \\
S\end{array}$ & $\begin{array}{c}16 \\
R\end{array}$ & $\begin{array}{l}1 \\
S\end{array}$ & $\begin{array}{c}12 \\
\text { NS }\end{array}$ \\
\hline
\end{tabular}

AN: amikacin; ATM: aztreonam; CAZ: ceftazidime; CIP: ciprofloxacin; COL: colistin; CTX: cefotaxime; ERT: ertapenem; FEP: cefepime;

GM: gentamicin; IMP: imipenem; MEM: meropenem; TIG: tigecycline.

a $\mathrm{R}$ : resistant; NS: non-susceptible; S: sensitive. 
The fact that the patient in this report was colonised by a KPC-positive $K$. pneumoniae in his gastrointestinal tract suggests the in vivo transfer of bla $a_{\mathrm{KPC}-2}$ to $C$. koseri. Given that $K$. pneumoniae was not isolated from bronchoalveolar lavage cultures, it is most likely that the transmission could have occurred in the gastrointestinal tract. Bronchoalveolar lavage and sputum cultures during the patient's first hospitalisation did not reveal the presence of $C$. koseri or $K$. pneumoniae or other bacteria. It could be hypothesised that, during his first hospitalisation, he was colonised in his gastrointestinal tract by a $K$. pneumoniae of ST147, which predominates in this setting (unpublished data). A probable scenario is that the $b a_{\mathrm{KPC} .2}$ gene was transmitted from $K$. pneumoniae to $C$. koseri, since both are part of the gastrointestinal flora. Identification of a KPC-encoding C. koseri strain from faecal samples would provide support for this hypothesis. No such strain was isolated, but given the low minimum inhibitory concentration of imipenem against the $C$. koseri isolate, a likely explanation is that the imipenem concentration used for screening of faecal samples $(1 \mathrm{mg} / \mathrm{L})$ suppressed the growth of $C$. koseri.

Rapid spread of carbapenemase-producing Enterobacteriaceae is a serious concern in clinical patient care in Greece. The finding of an additional enterobacterial species, C. koseri, producing KPC underscores the increasing clinical importance of carbapenemase-positive microorganisms in this country.

\section{*Authors' correction:}

At the request of the authors, the word 'anaerobic' was deleted from the sentence ' $C$. koseri, an environmental, anaerobic, Gram-negative bacterium, is occasionally found as a coloniser of the human gastrointestinal tract'. This change was made on 24 October 2011.

\section{References}

1. Gatselis N, Malli E, Papadamou G, Petinaki E, Dalekos GN. Direct detection of Cardiobacterium hominis in serum from a patient with infective endocarditis by broad-range bacterial PCR. J Clin Microbiol. 2006;44(2):669-72.

2. Cai T, Giubilei G, Vichi F, Farina U, Costanzi A, Bartoletti R. A rare case of lethal retroperitoneal abscess caused by Citrobacter koseri. Urol Int. 2007;79(4):364-6.

3. Harada S, Ishii Y, Saga T, Tateda K, Yamaguchi K. Chromosomally encoded blaCMY-2 located on a novel SXT/ R391-related integrating conjugative element in a Proteus mirabilis clinical isolate. Antimicrob Agents Chemother. 2010;54(9):3545-50.

4. Kanamori H, Yano H, Hirakata Y, Endo S, Arai K, Ogawa M, et al. High prevalence of extended-spectrum \{beta\}-lactamases and qnr determinants in Citrobacter species from Japan: dissemination of CTX-M-2. J Antimicrob Chemother. 2011; 66(10):2255-62.

5. Tian GB, Adams-Haduch JM, Qureshi ZA, Wang HN, Doi Y. CTX-M-35 extended-spectrum beta-lactamase conferring ceftazidime resistance in Citrobacter koseri. Int J Antimicrob Agents. 2010;35(4):412-3.

6. Perilli M, Mugnaioli C, Luzzaro F, Fiore M, Stefani S, Rossolini GM, et al. Novel TEM-type extended-spectrum beta-lactamase, TEM-134, in a Citrobacter koseri clinical isolate. Antimicrob Agents Chemother. 2005;49(4):1564-6.

7. Castanheira M, Debbia E, Marchese A, Jones RN. Emergence of a plasmid mediated bla (VIM-1) in Citrobacter koseri: report from the SENTRY Antimicrobial Surveillance Program (Italy). J Chemother. 2009;21(1):98-100.

8. European Committee on Antimicrobial Susceptibility Testing (EUCAST). Clinical breakpoints. EUCAST. [Accessed 20 Jan 2011]. Available from: http://www.eucast.org/ clinical_breakpoints

9. Giske CG, Gezelius L, Samuelsen $\emptyset$, Warner M, Sundsfjord A, Woodford N. A sensitive and specific phenotypic assay for detection of metallo- $\beta$-lactamases and KPC in Klebsiella pneumoniae with the use of meropenem disks supplemented with aminophenylboronic acid, dipicolinic acid and cloxacillin. Clin Microbiol Infect. 2011;17(4):552-6.

10. Doi Y, Paterson DL, Adams-Haduch JM, Sidjabat HE, O'Keefe A, Endimiani A, et al. Reduced susceptibility to cefepime among Escherichia coli clinical isolates producing novel variants of CMY-2 $\beta$-lactamase. Antimicrob Agents Chemother. 2009;53(7):3159-61.

11. Tzelepi E, Magana Ch, Platsouka E, Sofianou D, Paniara O, Legakis NJ, et al. Extended-spectrum $\beta$-lactamase types in Klebsiella pneumoniae and Escherichia coli in two Greek hospitals. Int J Antimicrob Agents. 2003;21(3):285-8.

12. Papagiannitsis CC, Giakkoupi P, Vatopoulos AC, Tryfinopoulou K, Miriagou V, Tzouvelekis LS. Emergence of Klebsiella pneumoniae of a novel sequence type (ST383) producing VIM4 , KPC-2 and CMY-4 $\beta$-lactamases. Int J Antimicrob Agents. 2010;36(6):573-4.

13. Diancourt L, Passet V, Verhoef J, Grimont PA, Brisse S. Multilocus sequence typing of Klebsiella pneumoniae nosocomial isolates. J Clin Microbiol. 2005;43(8):4178-82.

14. Rasheed JK, Biddle JW, Anderson KF, Washer L, Chenoweth C, Perrin J, et al. Detection of the Klebsiella pneumoniae carbapenemase type 2 carbapenem-hydrolyzing enzyme in clinical isolates of Citrobacter freundii and $\mathrm{K}$. oxytoca carrying a common plasmid. J Clin Microbiol. 2008;46(6):2066-9.

15. Cai JC, Zhou HW, Zhang R, Chen GX. Emergence of Serratia marcescens, Klebsiella pneumoniae, and Escherichia coli isolates possessing the plasmid-mediated carbapenemhydrolyzing $\beta$-lactamase KPC-2 in intensive care units of a Chinese hospital. Antimicrob Agents Chemother. 2008;52(6):2014-18.

16. Petrella S, Ziental-Gelus N, Mayer C, Renard M, Jarlier $V$, Sougakoff W. Genetic and structural insights into the dissemination potential of the extremely broad-spectrum class A $\beta$-lactamase KPC-2 identified in an Escherichia coli strain and an Enterobacter cloacae strain isolated from the same patient in France. Antimicrob Agents Chemother. 2008;52(10):3725-36.

17. Vatopoulos A. High rates of metallo-beta-lactamaseproducing Klebsiella pneumonia in Greece - a review of the current evidence. Euro Surveill. 2008;13(4):pii=8023. Available from: http://www.eurosurveillance.org/ViewArticle. aspx?Articleld $=8023$

18. Maltezou HC, Giakkoupi P, Maragos A, Bolikas M, Raftopoulos $\mathrm{V}$, Papahatzaki $\mathrm{H}$, et al. Outbreak of infections due to KPC2-producing Klebsiella pneumoniae in a hospital in Crete (Greece). J Infect. 2009;58(3):213-9.

19. Barbier F, Ruppé E, Giakkoupi P, Wildenberg L, Lucet J, Vatopoulos A, et al. Genesis of a KPC-producing Klebsiella pneumoniae after in vivo transfer from an imported Greek strain. Euro Surveill. 2010;15(1):pii=19457. Available from: http://www.eurosurveillance.org/ViewArticle. aspx?Articleld $=19457$

20. Giakkoupi P, Papagiannitsis CC, Miriagou V, Pappa O, Polemis $M$, Tryfinopoulou K, et al. An update of the evolving epidemic of blaKPC-2-carrying Klebsiella pneumoniae in Greece (20092010). J Antimicrob Chemother. 2011;66(7):1510-3. 